\title{
EDITORIAL
}

\section{Consolidating the Journal (JNSF) Policy}

The National Science Foundation (NSF) of Sri Lanka currently publishes four issues of its Journal annually. Its policy is to publish articles in all fields of science and technology (S\&T) under eleven categories. These include Research Articles, Short Communications, Reviews, Feature Articles, General Articles, Research Notes, Correspondence, Research News, Opinions, Book Reviews as well as Commentaries and Notes.

Details about these categories are given at the end of each issue of the Journal. This is to remind and encourage the scientific community to submit articles in these categories.

An analysis of the number of publications in the different categories in the 2014 issues of the JNSF shows that the majority are in the category of research articles amounting to $80 \%$. The next highest number of publications amounting to about $17 \%$ is in the category of short communications. The balance of little over $2 \%$ is in the category of research notes. Publications in the categories of short communications and research notes also deal with results of research studies conducted by the scientists. There are no publications in any of the other categories. Although the number of research articles published is satisfactory, the numbers published in the other ten categories certainly are not satisfactory and it is hoped that the scientists will endeavour to improve this situation in the future.

This scenario raises several questions in one's mind. Why do scientists publish mainly research articles that disseminate their research findings? Is it due to added incentives to the scientists that stem from research articles as compared to the articles in the other categories? Even if this is the case, the scientists have the responsibility to contribute publications in the other categories which serve a wider community. The policy of the Journal is to publish under eleven categories, which encourages the S\&T community to disseminate more useful information to the scientific community and thereby enhance the quality of the journal.

Over $95 \%$ of the publications in the four issues of 2014, have been authored by teams of researchers numbering 02 to 07 . Less than $5 \%$ of the articles have been published by sole authors. The high percentage of multiple authorship, in my opinion, is a praiseworthy feature where teams of research scientists have worked together. These teams of authors have members from different disciplines and different institutions with the university academics playing a major role. Some teams included scientists from only local institutions while some other teams included both local and foreign scientists from local and foreign institutions. It is necessary to further develop these types of collaboration in research studies particularly when seeking answers to complex problems faced by the society.

In the context of Sri Lanka, the country faces many such complex problems for which the scientists must endeavor to find solutions. These complex problems, to name a few are management of natural disasters, climate change impacts, environmental challenges, health problems such as Dengue outbreaks and the chronic kidney disease of unknown etiology (CKDu). In all these cases, solutions cannot be found by working within narrow and isolated disciplines. Nature does not operate within confines of disciplines. We scientists need to be guided accordingly. Collaboration among scientists with specific expertise in different disciplines from different institutions in the country and foreign institutions where necessary, is surely the approach that must be nurtured.

The western scientific tradition resulted in the emergence of new disciplines and sub-disciplines with well-defined boundaries and areas. This no doubt led to 
the development of vast amounts of new knowledge in these isolated disciplines. However, it has been seen that collaboration and communication between and among the experts in the different disciplines do not occur easily. Further, in modern society the development of the cultures of science and humanities have also hindered to a large extent the effective solution of societal problems. It has to be recognized that there is an urgent need for integrating these cultures and the scientists have a large responsibility towards achieving this. 